\title{
Protection Mechanisms for Optical WDM Networks based on Wavelength Converter Multiplexing and Backup Path Relocation Techniques
}

\author{
Sunil Gowda* and Krishna M. Sivalingam ${ }^{\dagger}$ \\ * Expedia.com, Seattle, WA \\ $\dagger$ University of Maryland Baltimore County (UMBC), Dept. of CSEE, Baltimore, MD 21250
}

\begin{abstract}
This paper studies the problem of designing survivable optical wavelength division multiplexed (WDM) networks. A wavelength-routed wide area backbone network supporting circuit-switched traffic is considered. This paper also considers the use of optical wavelength conversion technology which has been shown to help improve network performance. However, wavelength conversion is still an expensive technology and using optical conversion could potentially result in signal quality degradation.

In survivable networks, protection against failures is provided using backup paths that are determined when a session is established. In this paper, we present three primary and backup route computation mechanisms that attempt to improve overall network performance compared to existing solutions. One of the key design goals is to reduce the number of required converters per node. First, we present a routing algorithm, termed conversion free primary routing (CFPR) that computes primary paths without wavelength conversion, as far as possible. Next, we present a converter multiplexing technique that is used to share wavelength converters among multiple backup paths. This significantly reduces the number of connections blocked due to wavelength converter unavailability and reduces the number of wavelength converters required at each node, thus reducing system cost. Finally, we propose a backup path relocation scheme that migrates existing backup paths, whenever needed, to accommodate more primary paths and also to obtain primary routes with fewer hops. This is done to improve network utilization and reduce blocking probability. The proposed techniques are analyzed in detail using a discrete-event simulation model. The results show that significant reduction in blocking probability is possible with the proposed mechanisms. The number of converters required at each node to achieve a given blocking probability is also seen to be four times lower, compared to existing architectures based on static shortest path routing.
\end{abstract}

\section{INTRODUCTION}

The emergence of broadband multimedia services and distributed applications has resulted in an exponential growth in bandwidth requirements. Optical wavelength-division multiplexed (WDM) networking technology offers a promising solution to this huge bandwidth requirement foreseen for the next generation Internet [1], [2]. WDM technology bridges the gap between the optical fiber bandwidth and the peak electronic data rates, in the gigabits per second range, by partitioning the gross bandwidth into a number of smaller bandwidth channels manageable by electronic components. This paper considers routing algorithm design for a survivable all-optical meshtopology based wide area backbone network (WAN). The network consists of optical switches interconnected by multiwavelength WDM links.

A circuit switched network is studied with all-optical paths being established between the end nodes. An all-optical path or lightpath consists of a set of intermediate links, with typically one wavelength allocated to a given session request. A connection request is considered blocked if sufficient free resources are not available on all links of the path. In the absence of wavelength conversion [3], a lightpath must occupy the same wavelength on all the traversed links. If the wavelength routers possess wavelength conversion capability, an optical signal may be converted from one wavelength to another wavelength. Thus the blocking probability is reduced, as a lightpath no longer has the wavelength continuity constraint. However, wavelength converters are expensive devices and hence should be used sparingly; also, signal quality degradation is possible, dictating a limit on the number of conversions per lightpath.

Network monitoring statistics show that failures are not an uncommon occurrence in backbone networks [4], [5]. Hence, fault-tolerance (or survivability) is an important consideration for such high capacity networks, since failures may result in significant degradation of network performance [6]. Survivability is introduced in an optical network by provisioning both a primary path and a backup path for each connection that is setup. The data is typically transmitted only on the primary path during normal network operation and switched to the backup path when failure occurs.

This paper presents three different mechanisms to help improve network performance for survivable WDM mesh networks. Typically, routing for mesh networks is based on pre-computed static routes (e.g. shortest hop-count). We have presented, in earlier work, the benefits of dynamic route computation based on WDM-specific link information [7], [8]. We present in this paper an enhancement of this dynamic route computation mechanism. We propose a routing scheme for primary paths, termed conversion free primary routing (CFPR), that computes wavelength conversion free primary paths. The objective is to eliminate conversion delay, possible signal degradation and also to reduce the number of converters needed in the network, thereby reducing the cost.

We then propose a converter multiplexing technique that allows wavelength converters to be shared among multiple backup paths. This significantly reduces the number of connec- 
tions blocked due to wavelength converter unavailability and also reduces the number of wavelength converters required at each node.

We next present a backup path relocation scheme that migrates existing backup paths whenever necessary. This helps in routing a new primary path over a shorter path, and is also helpful in computing conversion-free primary paths. As there is no active transmission on a backup path, there is no disruption in service during the relocation.

The performance of the network incorporating these mechanisms is studied using discrete event simulation. The results based on a 24-node topology with 16 wavelengths per link indicate that the proposed algorithms reduce the required number of wavelength converters at each node by $75 \%$. The overall blocking probability reduction ranged from 60 to nearly $100 \%$ with the proposed schemes. The number of primary paths undergoing conversion is also significantly reduced while the overhead introduced is negligible. The overall network blocking probability was reduced by up to an order of magnitude, with higher improvements seen for low to moderate loads.

The rest of the paper is organized as follows. Section II presents relevant background material on WDM networks and protection techniques. Section III presents the details of the three proposed mechanisms. The performance analysis of the proposed techniques is presented in Section IV. Section V concludes the paper.

\section{BACKGROUND}

This section presents relevant background information on routing and wavelength assignment (RWA) algorithms, protection mechanisms, and on wavelength router architectures that incorporate conversion.

RWA problem: This paper considers a dynamic network model where connection (or session) requests arrive based on a stochastic process. Each request specifies the source and destination nodes and the bandwidth required (here, we assume that each session requires one wavelength). Each session is assigned a lightpath on a specific path/wavelength combination for its entire duration. The problem of determining the end-toend route and the specific wavelength on each link is referred to as the routing and wavelength assignment (RWA) problem. A survey of some of the solutions for this problem may be found in [9].

The different approaches can be broadly classified as static or dynamic routing schemes. In the static routing scheme, the route between each node pair is fixed and when a request arrives, the specific wavelength is determined dynamically based on some wavelength reservation protocol. In the dynamic routing scheme, the route is computed based on the current network status as in [7], [8]. WDM-specific link information such as available wavelengths and total wavelengths are used to compute link weights that are utilized in Dijkstra's shortest-cost path computation algorithm. In this paper, we use a dynamic route computation technique, based on perwavelength status information as explained later. Routes are computed individually on each wavelength plane, based on an augmented graph method presented in [10].

Wavelength router architectures: In a network without wavelength conversion, connections are often blocked since the same wavelength is not available on all links of the path. This constraint can be eliminated when wavelength conversion is available. Here, the lightpath can use different wavelengths on different links of the path. The disadvantages of conversion are its high cost, lack of availability in high commercial volumes and potential signal degradation with multiple conversions on a lightpath.

Optical wavelength conversion techniques have been studied from various perspectives: conversion-based router architectures [11], converter placement [12], converter allocation [13], and sparse wavelength conversion [14], [15]. In this paper, we focus on minimizing the usage of wavelength conversion for primary paths and thus reduce the number of converters required.

In a wavelength convertible router, wavelength converters are typically located between the output of the switching fabric and the input of the multiplexer on the outgoing link. Three different architectures have been proposed for a wavelength convertible switch: dedicated, share-per-link and share-pernode [11].

In a dedicated wavelength-convertible switch architecture, a wavelength converter is available at each output port of the switching fabric. Thus, up to $W$ converters are available per output link, where $W$ is the number of wavelengths per link. This completely eliminates the blocking of connection requests due to unavailability of wavelength converters. However, the disadvantages are the large number of converters required and the potentially low utilization of each converter.

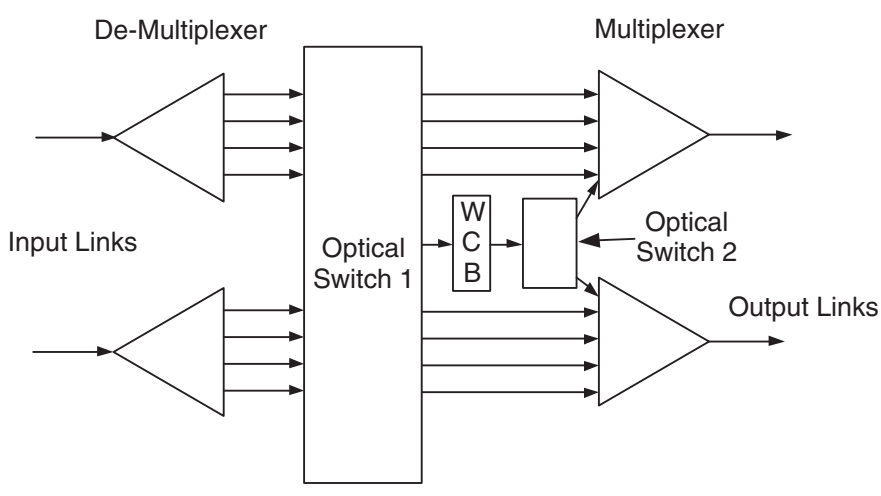

Fig. 1. Share-per-node wavelength convertible switch architecture.

In the share-per-node architecture, a single wavelength converter bank (WCB) is provided for the entire router, as shown in Figure 1. A lightpath signal requiring wavelength conversion is directed through a port leading to the bank of wavelength converters. The advantages of this architecture are lower cost due to small number of wavelength converters per node and high converter utilization. The disadvantages are higher switching complexity due to the introduction of the 
second switch, and blocking of connections due to converter unavailability, as typically fewer number of converters are provided in the share-per-node architecture compared to the dedicated architecture.

The share-per-link wavelength convertible switch architecture has a bank of converters for each link. The performance and cost of this architecture lie between that of the dedicated and share-per-node schemes.

Protection in mesh-topology WDM networks: This section summarizes some of the previous work done in the area of recovery mechanisms for mesh-topology based optical networks. Network failures are typically categorized as either link failures or node failures. Link failures may be due to fiber cuts, transceiver failures or other reasons, and require rerouting of lightpaths on the affected link. When a node fails, the affected lightpaths have to be handled by the other nodes. Other types of component failures are also possible [16], but are not addressed in this paper. In this paper, we focus on the handling of link failures.

Recovery is typically accomplished by identifying an alternate or backup path, when the primary path for a session fails. The failure recovery mechanisms are classified as protection (proactive) and restoration (reactive) mechanisms. [4]. In protection mechanisms, the recovery (or backup) paths are identified and resources allocated (with possible backup sharing) in advance; whereas in restoration mechanisms, the paths are set up only after the failure occurs. Protection mechanisms have a lower recovery time, but require redundant spare capacity. Restoration mechanisms are more efficient in resource utilization, but take much longer to restore the connection and cannot offer $100 \%$ restoration guarantee.

Protection and restoration mechanisms are both further classified as link-level and path-level mechanisms. In linklevel mechanisms, failures are handled by re-routing traffic around the failed link. In path-level mechanisms, a link or node-disjoint backup path is computed between the end-nodes of each affected lightpath. Link level mechanisms are faster and require fewer control messages, but have higher resource requirements. Path level mechanisms have a higher restoration time and higher control overhead, but are more efficient in terms of resource utilization.

Protection mechanisms may further be classified as dedicated and shared protection schemes [17]. In the dedicated protection scheme, termed 1:1 protection, a dedicated backup path is established for each individual connection. In shared protection schemes, termed 1:M protection, a wavelength (or link) may be shared among multiple backup paths provided they are both never activated simultaneously. This is also referred to as backup path multiplexing. This technique significantly reduces the blocking probability, while offering $100 \%$ restoration guarantee in the case of single link failures.

A summary of solutions for recovery mechanisms for ring and mesh networks may be found in [18], [19]. A survey of protection mechanisms from an implementation perspective appears in [4]. Also, most of the earlier research has studied single link (or node) failures at a given time instant. Recent research has started to address the dual failure problem [20][23].

Lightpath migration: Migration of lightpaths onto new paths to accommodate other connections has been studied in [24], [25]. A virtual topology reconfiguration scheme to adapt to changing traffic patterns has been modeled as an Integer Linear Programming (ILP) formulation in [26]. However, these approaches require the lightpaths to be torn down and reestablished on the new paths. During the reconfiguration phase, the transmission on the current path has to be terminated. A backup path migration scheme has been proposed in [27] where backup paths are migrated to paths selected from a set of $k$ precomputed paths. As there are no ongoing transmissions on the backup paths, they do not require termination of ongoing transmission. However, it appears that the drop in network performance as reported in the results is a result of using the static set of routes. Migration of backup paths as part of a medium term network reconfiguration has been studied in [5]. Here, re-routing is done on a set of backup paths at periodic intervals or as necessary.

\section{PRoposed Mechanisms}

This section presents the network architecture studied and the details of the proposed mechanisms.

\section{A. Network Architecture}

The network architecture studied is the mesh-topology based wide-area wavelength routed network, using wavelength conversion. The specific details are listed below:

1. A dynamic routing mechanism is used, where the shortest path between each node pair is computed periodically based on the current network status [7], [8]. We assume that a distributed link state routing protocol such as OSPF is used, with topology information being periodically exchanged among the nodes.

2. Path-level protection (i.e. proactive) mechanisms with both dedication protection and shared protection (backup path multiplexing) are considered.

3. A wavelength router architecture based on the share-pernode wavelength converter configuration is used. This is chosen since it offers the best cost to performance ratio.

4. Connections are assumed to be blocked only due to unavailability of free wavelengths and/or wavelength converters.

The design goals of the proposed mechanisms are to improve performance by reducing the number of connections blocked due to wavelength converter unavailability, and to reduce cost by lowering the number of converters required per node. In order to achieve this, we first propose the ConversionFree Primary Routing (CFPR) scheme for routing primary connections. We choose to route primary connections on conversion free paths, for reasons explained later. If conversion free paths are not available, the hop-count based shortest path routing algorithm is used to route the connection. Such a path will require wavelength conversion and could experience blocking if wavelength converters are not available. We then 
propose the converter multiplexing technique that allows sharing of wavelength converters among backup paths. The backup path are routed using a static shortest path routing scheme, after eliminating the links used by the corresponding primary. We also present a backup path relocation policy that is used to migrate backup connections onto new routes to accommodate requests that would otherwise have been rejected. The three proposed mechanisms are presented in the following sections.

\section{B. Conversion Free Primary Routing (CFPR) Technique}

In typical protection schemes, the route for the primary and backup paths are pre-determined using a hop-count based route computation algorithm [5], [17]. In this paper, paths for the primary and backup connections are determined using two different routing schemes.

Since primary paths require dedicated resources, including wavelength converters, it is preferable to route primary connections on conversion-free paths. Wavelength conversion may also possibly degrade the signal quality and introduce a conversion delay. Thus, one objective is to avoid wavelength conversion while routing primary connections, as far as possible. This is achieved using the Conversion-Free Primary Routing (CFPR) described below.

For a wavelength convertible network, typically the multiple layers representing individual wavelength planes are interconnected using edges between corresponding nodes of the different wavelength layers [10]. The CFPR algorithm models the network using a multi-layered graph although the network has wavelength conversion capability. Based on the physical topology, a modified network graph is constructed as follows. For each wavelength plane, the nodes are the physical nodes and an edge exists between two nodes if the wavelength is either not allocated or is reserved for a backup path. This is denoted as $F_{i, j}^{w}=0$, if wavelength $w$ on link $(i, j)$ is assigned to a primary path, and 1 , if the wavelength is either not allocated or is reserved for some backup path(s). The routes are then computed on each wavelength using Dijkstra's shortest path algorithm. Let $\mathbf{P}_{s d}^{w}$ denote the shortest path from node $s$ to node $d$ on wavelength $w ; \mathbf{P}_{s d}^{w}=\phi$ if no path is available on this wavelength. The routing scheme thus calculates up to $W$ paths, one on each wavelength. However, paths may not be available between each node pair on some wavelengths depending on the current wavelength usage status.

Let us consider the example shown in Figure 2. The shortest paths computed on each wavelength by CFPR between nodes 1 and 5 are shown here. On wavelength 0 , the route computed is $1-2-4-5$ and all links of this path are unoccupied. On wavelength 1 , the route computed is $1-3-5$, but link $3-5$ is currently occupied by some backup path $b$. Routing a connection on wavelength 1 requires $b$ to be relocated if possible. No path is available on wavelength 2 .

For a given request, the shortest path among the set of (up to) $W$ paths is selected as the route. However, the assigned wavelength may currently be occupied by backup paths on some links of the path as seen in the example earlier. These overlapping segments of the backup paths have to be
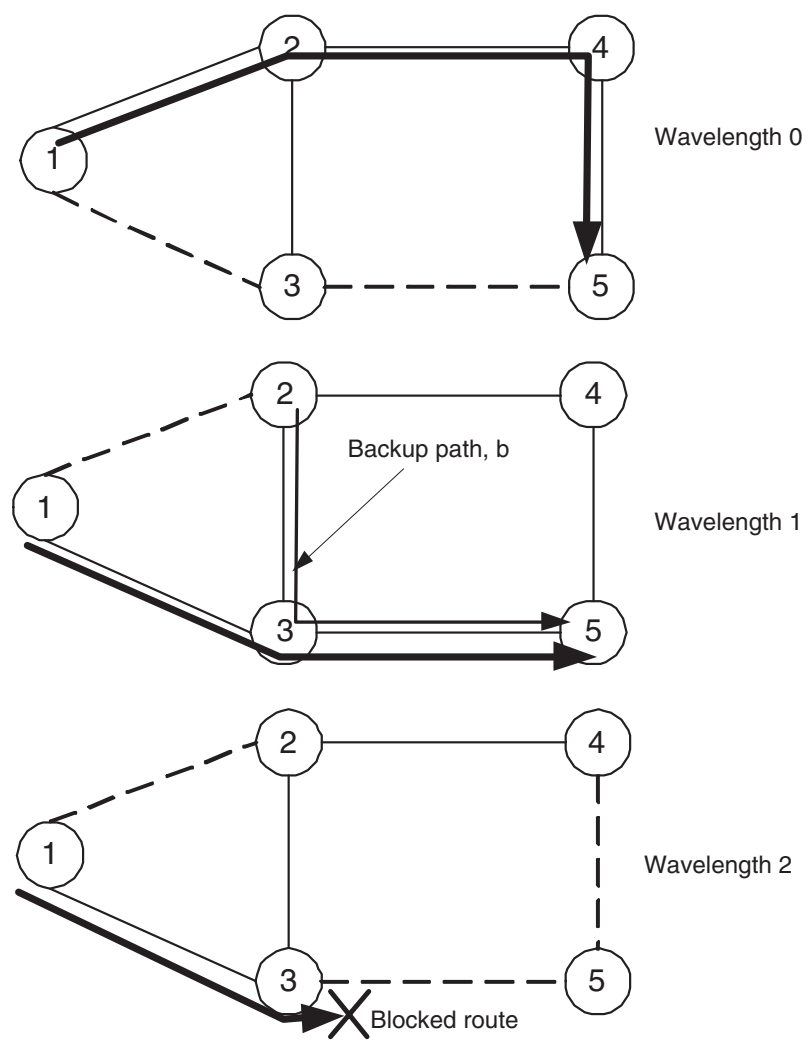

$$
\begin{aligned}
& \text { Unoccupied wavelengths/ } \\
& \text { Wavelengths occupied by } \\
& \text { backup paths }
\end{aligned}
$$

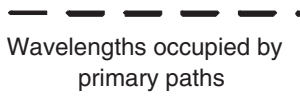

primary paths

Fig. 2. Conversion-Free Primary Routing.

relocated. An overlapping segment is defined as the part of the backup path occupying the wavelength assigned to the requested connection. The overlapping segments for path $p_{1}$ are marked in Figure 3. The relocation schemes discussed in the Section III-D may be used to relocate these overlapping segments.

The advantages of the CFPR mechanism are as follows. First, since each computed route is on a single wavelength, wavelength conversion is not required on these paths. This implies reduced conversion delays and potential converterinduced signal degradation. CFPR computes paths on each wavelength separately and hence alternate paths are computed if the shortest paths are blocked. Third, CFPR has lower computational complexity. For a network with $N$ nodes and $W$ wavelengths, computing shortest paths on each of the wavelength planes individually is a $O\left(N^{2} W\right)$ operation. In comparison, the modified graph in the conventional approach for routing in wavelength-convertible networks has $N W$ nodes and a complexity of $O\left(N^{2} W^{2}\right)$ [10].

Note that the relocation scheme is optional in this architecture. If we choose not to undergo the complexity of relocation, the CFPR and the converter multiplexing schemes alone may be used. 


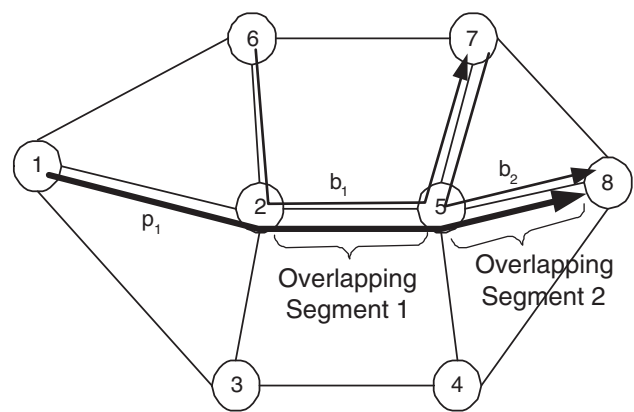

Fig. 3. The above figure shows a potential primary path with some of its component links overlapping existing backup paths.

\section{Converter Multiplexing}

Converter multiplexing is based on the backup path multiplexing mechanism, presented in Section II. Just as a wavelength is shared among multiple backup paths, a wavelength converter can be shared among multiple backup paths if their associated primary paths are routed on physically disjoint paths.

For example, consider the network shown in Figure 4 with 2 wavelengths on each link. Primary paths have been selected for node pairs $(1,8)$ and $(4,8)$. The primary paths are denoted as $p_{1}(1-6-7-8)$ and $p_{2}(4-8)$. The corresponding backup paths are $b_{1}(1-2-5-8)$ and $b_{2}(4-5-8)$ respectively. The backup paths are required to undergo wavelength conversion at node 5 . Since $p_{1}$ and $p_{2}$ are link disjoint, $b_{1}$ and $b_{2}$ can share a wavelength converter at node 5 . The converter multiplexing algorithm reduces the required number of used converters at node 5 from 2 to 1 . When scaled to a larger network with more than 100 wavelengths and thousands of established connections, the potential savings in the number of converters is higher. Also, note the number of backups that can share a converter is not limited in our architecture, provided each of the respective primary paths are link disjoint.

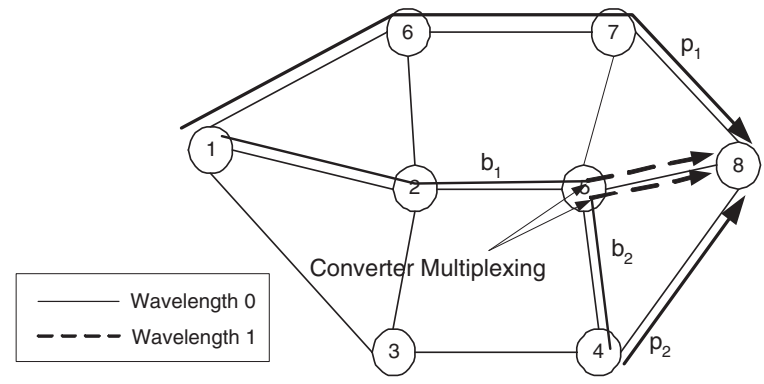

Fig. 4. Converter multiplexing between paths $b_{1}$ and $b_{2}$

In the backup path multiplexing architecture, the wavelengths are reserved during connection setup and are configured only after the failure occurs. Similarly, the converters are only reserved during the establishment of the backup paths, and are tuned to the required wavelength during the recovery process. A signaling mechanism similar to that studied in [28] may be used here. During establishment of the backup paths, CONV-RESV messages are sent to the intermediate nodes at which the backup path requires wavelength conversion. These nodes respond with a CONV-RESV-ACK if the request can be accepted. The source node completes the backup path selection only if all CONV-RESV-ACKS are received.

In order to ensure that converters are shared only among backup paths that have physically disjoint primary paths, a list of backup paths reserving the particular converter is maintained at each converter. Each node maintains a Wavelength Converter Status Table (WCST) shown in Table I. During establishment of the backup paths, a node receiving a CONVRESV message checks the WCST to select a converter. If a converter that can be reserved for the backup connection in consideration is available, a CONV-RESV-ACK message is sent to the source node. An entry in inserted into the WCST once the connection is established successfully. Otherwise, a CONV-RESV-NACK is sent. This process is similar to selecting a wavelength with backup path multiplexing, The first fit wavelength selection algorithm is used to select the wavelength converter as it requires the least number of converters.

\begin{tabular}{|c|c|c|c|c|}
\hline $\begin{array}{c}\text { Conv. } \\
\text { ID }\end{array}$ & $\begin{array}{c}\text { Conn. } \\
\text { ID }\end{array}$ & $\begin{array}{c}\text { Incoming } \\
\text { Port }\end{array}$ & $\begin{array}{c}\text { Outgoing } \\
\text { Port }\end{array}$ & Status \\
\hline 1 & 1 & $1-\lambda_{0}$ & $2-\lambda_{1}$ & reserved \\
\hline 1 & 2 & $3-\lambda_{2}$ & $5-\lambda_{0}$ & reserved \\
\hline 2 & - & - & - & free \\
\hline 3 & 4 & $1-\lambda_{0}$ & $4-\lambda_{1}$ & primary \\
\hline
\end{tabular}

TABLE I

WAVElength Converter Status TABle.

When network failure occurs and path recovery is initiated, CONV-SETUP messages are sent to the nodes to configure the reserved wavelength converter. Since control messages to intermediate nodes on the backup path are anyway required, our mechanism does not cause significant increase in control overhead or recovery time. Wavelength converter tuning delays are much lower (about 1 nanosecond [29]) compared to switch configuration delays (about 500 microseconds [28]).

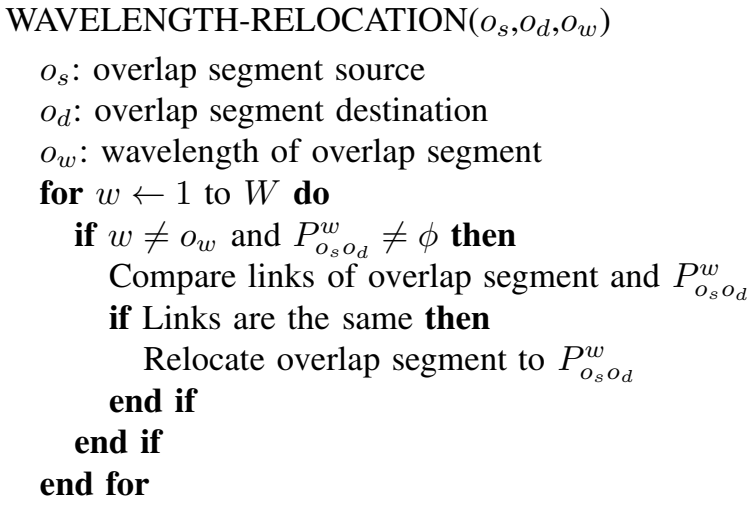

Algorithm 1: The Wavelength Relocation (WR) algorithm. 


\section{SEGMENT-RELOCATION $\left(o_{s}, o_{d}, o_{w}\right)$}

$O_{s}$ : overlap segment source

$o_{d}$ : overlap segment destination

$o_{w}$ : wavelength of overlap segment

for $w \leftarrow 1$ to $W$ do

if $w \neq o_{w}$ and $P_{o_{s} o_{d}}^{w} \neq \phi$ then

Relocate overlap segment on $P_{o_{s} o_{d}}^{w}$

$$
\text { end if }
$$

end for

Algorithm 2: The Segment Relocation (SR) algorithm.

\section{Backup Path Relocation}

In section III-B, we mentioned that existing backup paths may be relocated to provide potential performance improvements. Two such relocation schemes are proposed to migrate an overlapping backup segment: the wavelength relocation (WR) and the segment relocation (SR) techniques. In WR, a new wavelength is used for the overlapping segment, while the component links are unaltered. With SR, the overlapping segment can be relocated on a completely different path if required.

When a connection request arrives, the overlapping segments for the selected primary path are first determined. The backup paths are relocated only if all overlapping segments can be relocated successfully. If relocation fails for any of the overlapping segments, none of the overlapping segments are relocated. The CFPR algorithm then selects the path on the next candidate wavelength and continues as earlier.

Algorithm 1 presents the wavelength relocation algorithm. Consider the network shown in Figure 5 with 6 nodes and 3 wavelengths. For a connection request between nodes 1 and 6 , path $1-3-6$ on wavelength 0 is computed to be a candidate primary path. Assigning this path requires hop $1-3$ of backup path $b_{1}$ to be relocated. Wavelength 2 offers a path between nodes $1-3$. Thus, the overlapping segment is relocated to wavelength 2 , after reserving a converter at node 1 . The postrelocation state of path $b_{1}$ is shown in Figure 5 .

Algorithm 2 presents the wavelength relocation algorithm. Figure 5 illustrates segment relocation with an example. Here, backup path $b_{2}$ is relocated from path $2-3-6$ onto path $2-3-5-6$.

Between the two mechanisms, WR is simpler since the overlap segment's links are unchanged. Thus, control messages have to be sent only to the nodes on the overlapping segment about the reconfiguration. However, it is possible that a free wavelength may not be available on the same set of links and hence relocation may fail. In comparison, segment relocation considers a larger set of candidate paths and thus offers a higher probability of successful relocation. However, segment relocation incurs higher control overhead, as the resources on the overlapping segment have to be released and re-established on the new path. Segment relocation also consumes more resources due to potentially longer sub-paths.

\section{PERformance AnAlysis}

This section presents the performance analysis of the proposed mechanisms, based on a discrete-event simulation model.

\section{A. Simulation model}

We consider a dynamic network traffic model, where session requests arrive at a node according to a Poisson process with rate $\lambda$ and uniform destination node distribution. Session duration is exponentially distributed with a mean of $1 / \mu$. Each session request is assigned a wavelength. The traffic load per node, $L$, is defined as $\frac{\lambda}{\mu}$, and expressed in Erlangs.

The share-per-node wavelength convertible router architecture is considered, and all nodes are allocated an equal number of wavelength converters. The performance of both dedicated and shared protection schemes is studied. The single link failure model is assumed.

Simulations are performed for two networks; a 24-node ARPANET-like network [30] with 16 and 32 wavelengths on each link, and a random 50-node network with 32 wavelengths per link. The results for the 24-node, 16-wavelengths network are discussed here. The results for the other networks show similar trends.

The system parameters varied are: the load per node $(L)$ and the number of converters $(C)$ at each router. In the first set of results, $L$, is varied from 1.0 to 5.0 Erlang, for $C=8$. In the second set, $\mathrm{C}$ was varied as $C \in\{2-16\}$, for $L=3.0$ Erlangs per node.

Three different mechanisms are compared: the basic hopcount (HC) based shortest path routing algorithm, the CFPR routing algorithm with wavelength relocation, and CFPR routing algorithm with segment relocation. For each algorithm, results are reported for dedicated and shared protection. The notation $\mathrm{X}-\mathrm{Y}-\mathrm{Z}$ is used to specify an algorithm, where $X \in\{H C, C F P R\}$ denotes the routing algorithm; $Y \in$ $\{N R, W R, S R\}$ denotes no relocation, wavelength relocation and segment relocation respectively; and $Z \in\{D P, S P\}$ denotes dedicated and shared protection respectively.

The performance metrics presented are the blocking probability $\left(P_{b}\right)$, link and converter utilization, average hop count and statistics on backup path relocation. The performance was studied for various other metrics but are not presented here.

\section{B. Blocking Probability}

Network blocking probability is defined as the fraction of the total connection requests that are rejected. The goal of algorithm design is to minimize this metric.

Figure 6 presents the blocking probability $\left(P_{b}\right)$ performance with varying load and number of converters. In Figure 6(a), we observe that the performance of the architectures employing the proposed converter multiplexing and backup path relocation schemes perform significantly better than the basic scheme. For example, at $L=1$ and dedicated protection, CFPR-WR-DP and CFPR-SR-DP have $P_{b}=0$, while HCNR-DP experiences a blocking probability of about 0.0014 . 

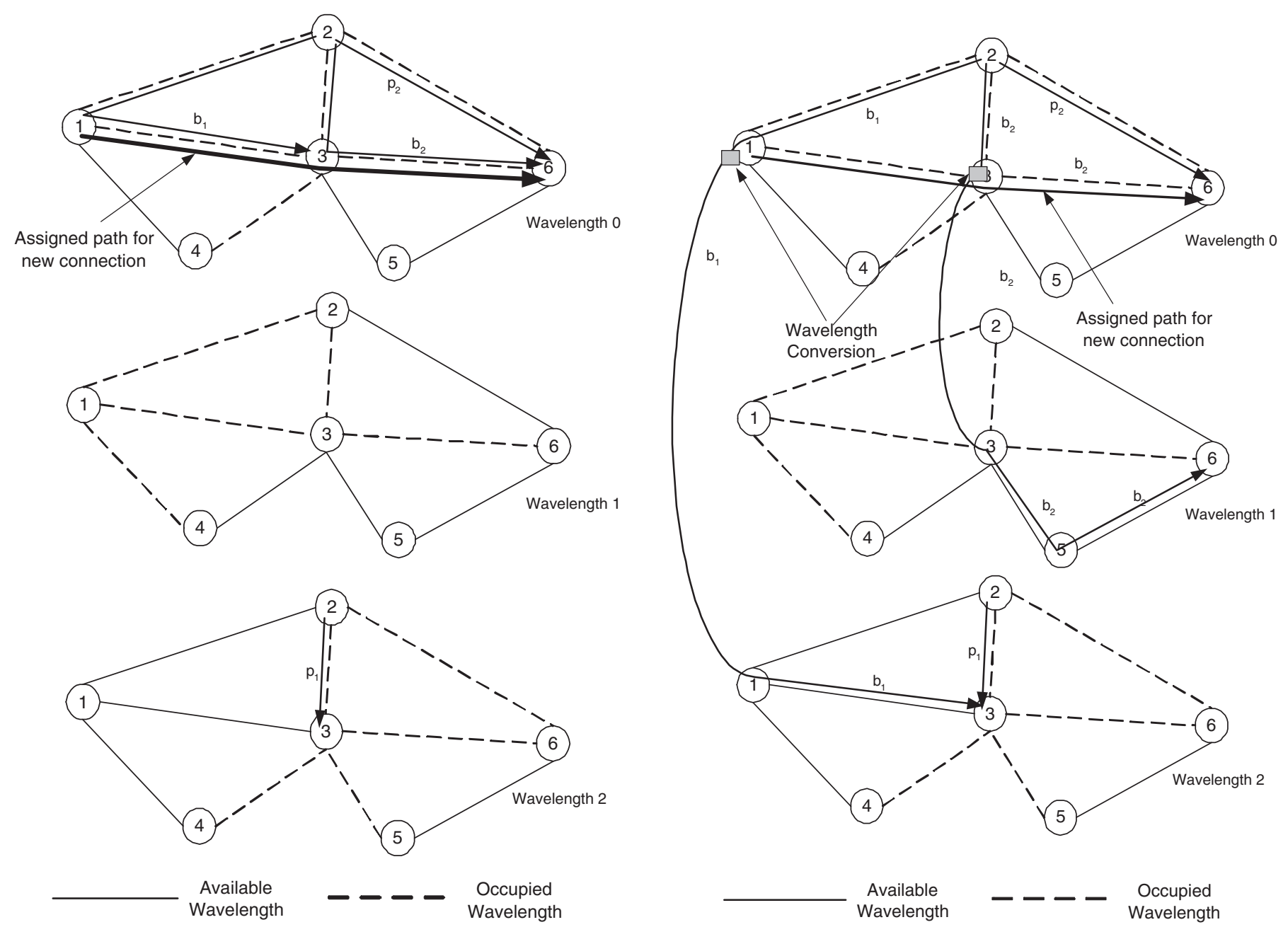

Fig. 5. Examples of the backup path relocation mechanisms. The left and right sets denote network states before and after relocation, respectively.

At $L=2$, the value of $P_{b}$ achieved by CFPR-WR-DP and CFPR-SR-DP is lower than that of the basic scheme by $95 \%$ and $96 \%$ respectively. The improvement is seen to diminish for higher loads, but is still better by about $60 \%$. For a target blocking probability lower than $10^{-1}$, the basic scheme can sustain loads up to 2.0 Erlang. In comparison, the proposed algorithms can handle loads up to 4.0 Erlang for this value of $P_{b}$.

Figure 6(b) demonstrates the reduction in the number of converters required per node. For an offered load of 3.0 Erlangs, the basic architecture needs a minimum of 16 converters to offer a blocking probability of less than $10^{-1}$. The new techniques offer the same performance for as few as 4 converters. We can also observe that having more than 8 converters, does not lower the blocking probability any further.

We also observe that segment relocation performs marginally better than wavelength relocation in the dedicated protection scheme and is identical in the shared protection scheme. This marginal improvement may not justify the increase in complexity and the involved control overhead. Hence, we conclude that the wavelength relocation scheme, combined with CFPR and converter multiplexing, is most suitable compared to the basic scheme.

\begin{tabular}{|c|c|c|c|}
\hline Scheme & Blocking Probability & $\% \mathrm{WU}$ & $\% \mathrm{CU}$ \\
\hline HC-NR-DP & 0.319 & $1 \%$ & $99 \%$ \\
\hline CFPR-WR-DP & 0.114 & $98 \%$ & $2 \%$ \\
\hline CFPR-SR-DP & 0.106 & $97 \%$ & $3 \%$ \\
\hline HC-NR-SP & 0.262 & $0 \%$ & $100 \%$ \\
\hline CFPR-WR-SP & 0.051 & $80 \%$ & $20 \%$ \\
\hline CFPR-SR-SP & 0.053 & $80 \%$ & $20 \%$ \\
\hline
\end{tabular}

TABLE II

PERCENTAGES OF CONNECTIONS BLOCKED DUE TO WAVELENGTH UNAVAILABILITY (\%WU) AND CONVERTER UNAVAILABILITY (\%CU).

We next analyze the different reasons for blocked connections. Table II lists the percentages of connections that were blocked due to wavelength unavailability (\%WU) and converter unavailability (\%CU) for $L=4.5$ and $C=8$. We observe that converter unavailability accounts for about $3 \%$ of 


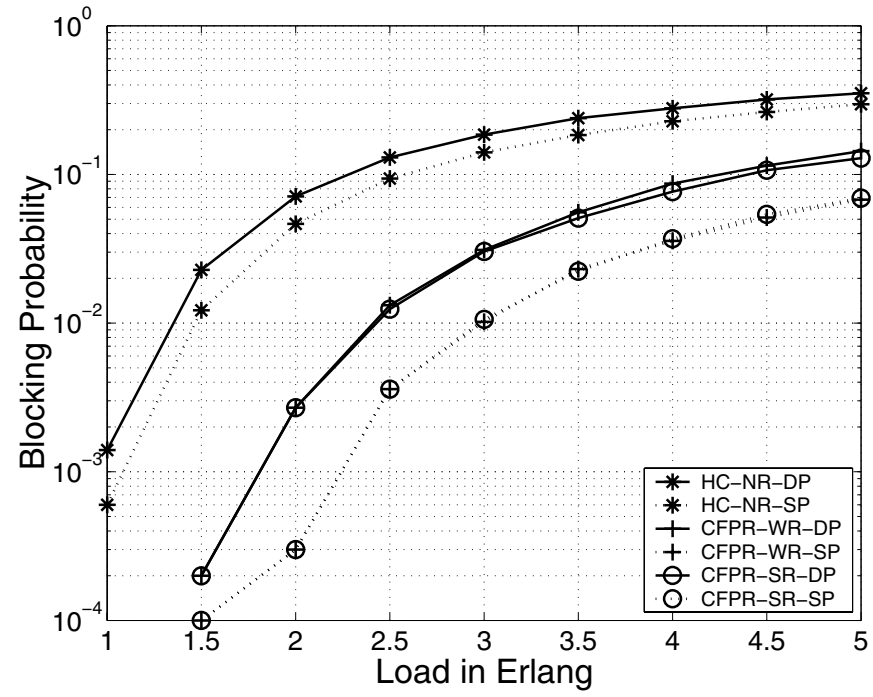

(a) Varying load

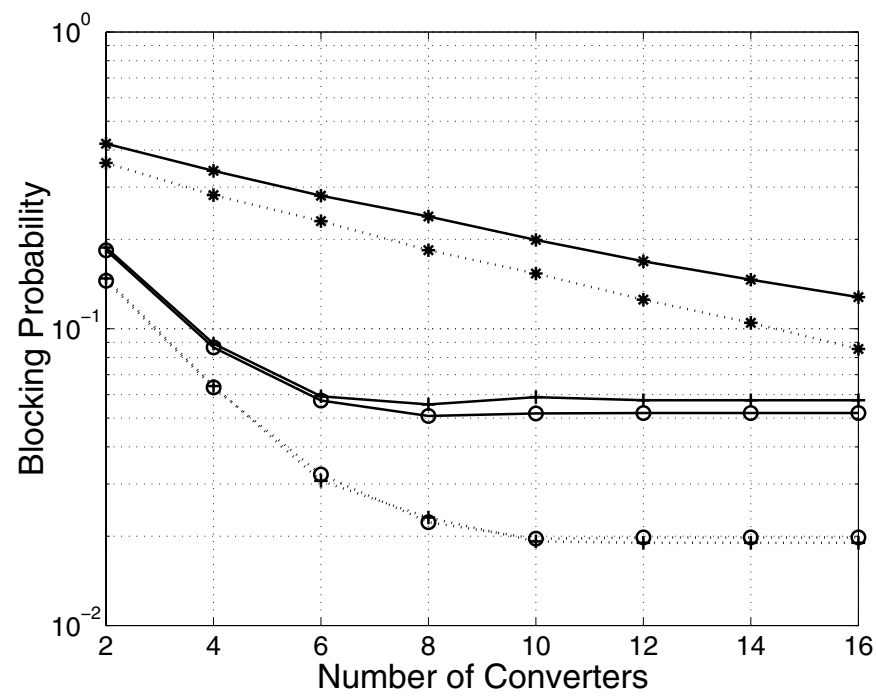

(b) Varying no. of converters

Fig. 6. Blocking probability performance varying $L$ and $C$ : (a) varying $L$ for $C=8$, and (b) varying $C \in\{4,8,12,16\}$ for $L=3$.

the blocked connections for the CFPR-based techniques for dedicated protection and about $20 \%$ for shared protection. In comparison, the same values for the basic schemes are $99 \%$ and $100 \%$. The proposed algorithms are thus able to accept more connections without requiring additional wavelength converters.

\section{Average hop count}

Figure 7 shows the average hop count of the primary paths for accepted connections. The basic scheme, which allocates dedicated wavelength converters for the primary path exhausts all the available converters as the network load increases. As fewer conversion-free paths are available between distant nodes, it can accept only shorter connections beyond a certain network load. For example, the average hop count for the basic

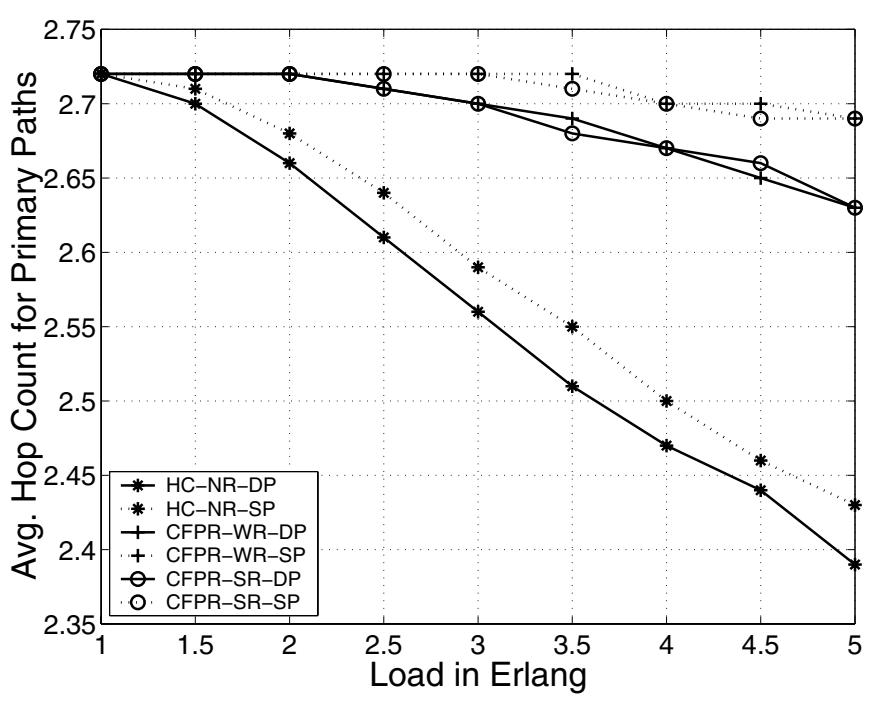

Fig. 7. Average hop count of primary paths.

scheme decreases from 2.7 at $L=1$ to 2.5 for $L=3.5$. In comparison, the converter multiplexing based algorithms, have a steady average hop count around 2.7. This is also the average distance between the nodes for the given topology.

Revenue: The blocking probability metric alone is not sufficient to compare the performance of two algorithms. Between two different algorithms, it is likely that one tends to accept more shorter hop connections, while another accepts more longer hop connections. Thus, the mere number of accepted connections does not provide for fair comparison of two algorithms. Hence, we define a revenue metric which is based on the number of hops routed. However, some algorithms may route connections on longer paths when a shorter path is available, to achieve load distribution. To avoid this problem, the revenue metric is defined as the shortest-hop count based on the static topology. A connection between two nodes which are 2 nodes apart on the static topology generates a revenue of 2 units, irrespective of whether the connection is actually routed on a 5-hop path or a 3-hop path. Thus, a higher value of overall revenue indicates that a given algorithm is able to accommodate both long and short-hop paths.

Figure 8 presents the revenue statistics for 100,000 connections requests for 2.0 Erlang and 4.0 Erlang. The proposed schemes are seen to provide higher revenue values compared to the basic scheme. For the basic scheme, the revenue drops as the load increases. This is because only the shorter connections are being accepted at high loads, while the longer ones are being dropped. The proposed algorithms on the other hand, show only a marginal drop in revenue. The marginal drop is attributed to a slight drop in the number of longer connections accepted.

\section{Conversion Statistics}

One of the primary objectives of the proposed algorithms was to provide wavelength-conversion free paths for the pri- 


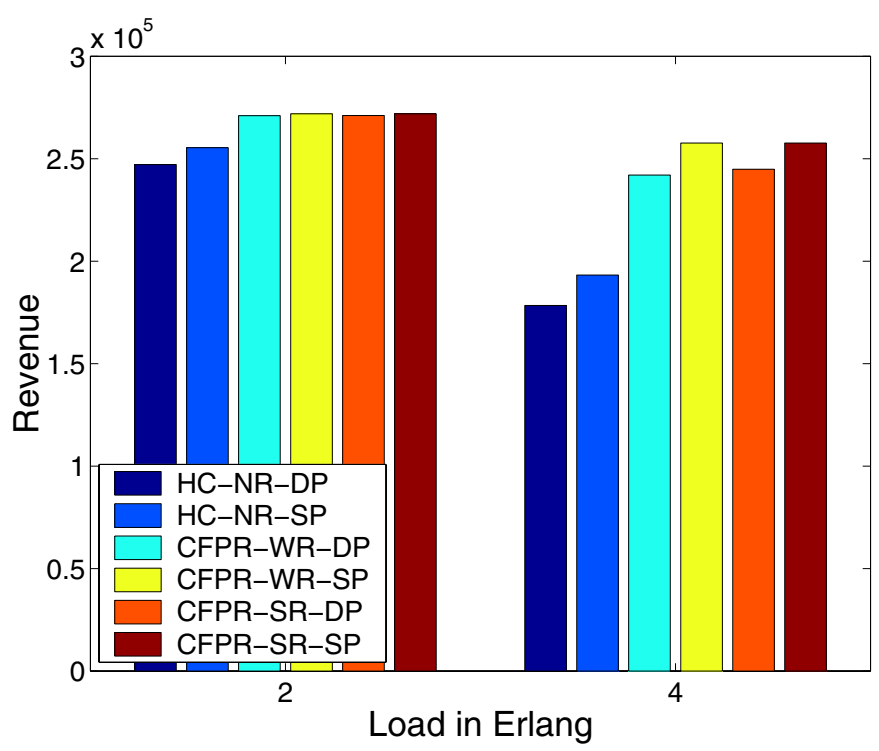

Fig. 8. Revenue generated for 100,000 connections.

mary paths. This section studies the performance of the proposed schemes from the viewpoint of wavelength conversion.

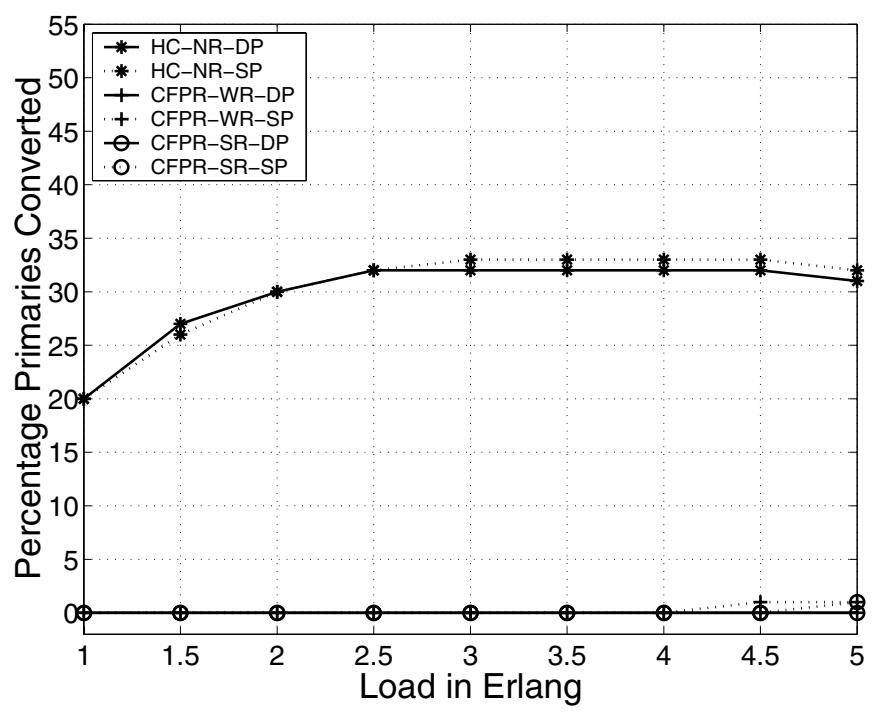

Fig. 9. Percentage of primary paths converted, varying $L$.

Figure 9 presents the percentage of primary paths requiring wavelength conversion for the different algorithms. As seen, around $30 \%$ of the connections routed by the basic routing scheme require at least one wavelength conversion on a primary path. In contrast, the proposed algorithms completely eliminate the need for wavelength conversion for the primary paths. This is a result of the the CFPR technique used to compute routes for primary paths. It is advantageous as alloptical wavelength conversion may degrade the signal quality, while opto-electronic wavelength conversion introduces delay. At higher loads, wavelength conversion-free paths are not available on any of the wavelengths and we see a few connections undergoing wavelength conversion.

\section{E. Relocation Statistics}

In order to understand the benefits of the relocation schemes, we studied the performance of systems with and without backup path relocation schemes. In the following discussion, REL and NO-REL denote the two schemes respectively. The blocking probability was seen to be lower, but not significantly, when relocation was deployed. But the more significant benefits were seen in the context of wavelength conversion. For a load of 7.5 Erlangs, $6 \%$ and $1 \%$ of the primary paths underwent wavelength conversion with NO-REL and REL respectively. Also, the percentage of connections blocked due to wavelength converter unavailability dropped from $33 \%$ in NO-REL to $3 \%$ in the REL scheme for a load of 3.5 Erlang with 4 converters at each node. The average hop count for the primary paths was also lower for REL indicating that primary paths were routed on shorter paths, thus consuming less resources and also leading to lower propagation delay.

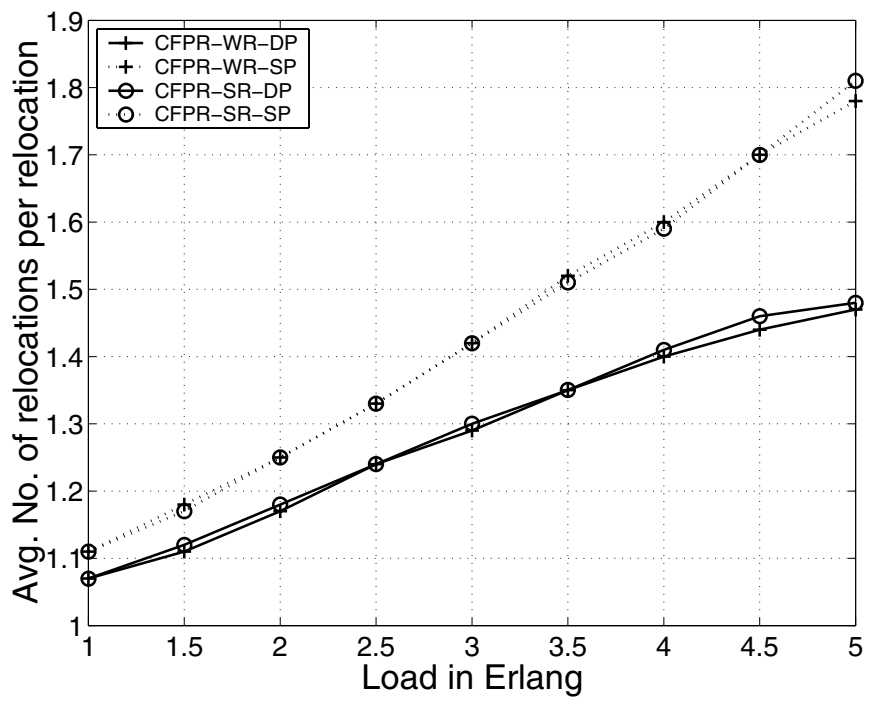

Fig. 10. Average number of relocations per instance.

Since relocations require extra computation and control message overhead, and it is essential to keep this low. The following experiment helps quantify this overhead in the studied network. Figure 10 presents the average number of backup paths that were relocated during establishment of primary paths. Only those connection requests that required relocations are considered while computing the average. At low loads, the average number of backup paths relocated is closer to 1.1; and increases to up to 1.9 (i.e. 2 paths) at higher loads. An average of 1.5 relocations is necessary at a load of 3.5 Erlang for the dedicated protection schemes. These results indicate that the backup relocation overhead is manageable, and only a reasonable number of relocations are necessary.

While we studied the use of backup path relocation with the goal of minimizing primary path conversion needs, relocation 
can be used for other reasons too. Consider a scenario where different network links have different quality-of-service properties, such as low interference, low distortion, higher security, higher reliability, etc. In this situation, primary paths may be routed on higher quality links by relocating backup paths onto lower quality links as and when required. This can be an issue for further research on this mechanism.

\section{Conclusions}

This paper studied routing algorithms and related mechanisms for survivable optical WDM mesh networks. Three different mechanisms were proposed and analyzed. In a dynamic connection oriented network, connection requests are blocked due to unavailability of free wavelengths and wavelength converters. The proposed converter multiplexing scheme significantly reduces the number of connections blocked due to unavailability of wavelength converters. It also required fewer number of wavelength converters at each node. The proposed CFPR routing algorithm significantly reduced the number of primary connections undergoing wavelength conversion. Two different backup path relocation mechanisms were also presented. The performance analysis showed that the combination of the proposed mechanisms results in substantial reduction in blocking probability compared to the basic routing scheme. Also, lower number of converters were required per node to achieve a target blocking probability. Between the backup relocation schemes, the additional overhead of using segment relocation compared to the wavelength relocation scheme did not result in much improvement. However, segment relocation can be used to allow primary connections to be routed on links offering better transmission quality.

\section{ACKNOWLEDGMENTS}

This research was partly supported by Cisco Systems, San Jose, CA. Part of the research was done while the authors were at Washington State University. Contact Author: Prof. Krishna Sivalingam (krishna@cs.umbc.edu).

\section{REFERENCES}

[1] P. Green, "Progress in optical networking," IEEE Communications Magazine, vol. 39, no. 1, pp. 54-61, Jan. 2001.

[2] K. Sivalingam and S. Subramaniam, Eds., Optical WDM Networks: Principles and Practice. Boston, MA: Kluwer Academic Publishers, 2000.

[3] B. Ramamurthy and B. Mukherjee, "Wavelength conversion in WDM networking," IEEE Journal on Selected Areas in Communications, vol. 16, no. 7, pp. 1061-1073, Sept. 1998.

[4] O. Gerstel and R. Ramaswami, "Optical Layer Survivability-An Implementation Perspective," IEEE Journal on Selected Areas in Communications, vol. 18, no. 10, pp. 1885-1923, Oct. 2000.

[5] M. Sridharan and A. Somani, "Revenue Maximization in Survivable WDM Networks," in Proc. SPIE OPTICOMM, Dallas, TX, Aug. 2000, pp. 291-302.

[6] A. Somani, "Understanding fault tolerance and reliability," IEEE Computer, vol. 30, no. 4, pp. 45-50, 1997.

[7] N. M. Bhide, K. M. Sivalingam, and T. Fabry-Asztalos, "Routing Mechanisms Employing Adaptive Weight Functions for Shortest Path Routing in Multi-Wavelength Optical WDM Networks," Journal of Photonic Network Communications, vol. 3, no. 3, pp. 227-236, July 2001.
[8] B. Wen and K. M. Sivalingam, "Routing, wavelength and time-slot assignment in time division multiplexed wavelength-routed optical WDM networks," in Proc. IEEE INFOCOM, New York, NY, June 2002.

[9] H. Zang, J. P. Jue, and B. Mukherjee, "A review of routing and wavelength assignment approaches for wavelength-routed optical WDM networks," Optical Networks Magazine, vol. 1, no. 1, pp. 47-60, Jan. 2000.

[10] I. Chlamtac, A. Farago, and T. Zhang, "Lightpath (Wavelength) Routing in Large WDM Networks," IEEE Journal on Selected Areas in Coтmunications, vol. 14, no. 5, pp. 909-913, June 1996.

[11] K.-C. Lee and V. Li, "A Wavelength-Convertible Optical Network," IEEE/OSA Journal of Lightwave Technology, vol. 11, no. 5, pp. 962970, May 1993.

[12] S. Subramaniam, M. Azizoglu, and A. K. Somani, "On optimal converter placement in wavelength-routed networks," IEEE/ACM Transactions on Networking, vol. 7, no. 5, pp. 754-766, Oct. 1999.

[13] G. Xiao and Y. Leung, "Algorithms for allocating wavelength converters in all-optical networks," IEEE/ACM Transactions on Networking, vol. 7, no. 4, pp. 545-557, Aug. 1999.

[14] S. Subramaniam, M. Azizoglu, and A. Somani, "Connectivity and Sparse Wavelength Conversion in Wavelength-Routing Networks," in Proc. IEEE INFOCOM, Mar. 1996, pp. 148-155.

[15] S. Subramaniam, M. Azizoglu, and A. K. Somani, "Connectivity and sparse wavelength conversion in wavelength-routing networks," IEEE/ACM Transactions on Networking, vol. 4, no. 4, pp. 544-557, Aug. 1997.

[16] H. Krishnamurthy, K. M. Sivalingam, and M. Mishra, "Restoration mechanisms based on tunable lasers for handling channel and link failures in optical WDM networks," in Proc. SPIE OPTICOMM, July 2002.

[17] S. Ramamurthy and B. Mukherjee, "Survivable WDM Mesh Networks Part 1 - Protection," in Proc. IEEE INFOCOM, vol. 2, New York, Mar. 1999, pp. 744-751.

[18] D. Zhou and S. Subramaniam, "Survivability in optical networks," IEEE Network Magazine, vol. 14, no. 6, pp. 16-23, Nov. 2000.

[19] S. Sengupta and R. Ramamurthy, "From Network Design to Dynamic Provisioning and Restoration in Optical XC Mesh Networks: An Architecture and Algorithm Overview," IEEE Network, pp. 46-54, Jul/Aug 2001.

[20] H. Choi, S. Subramaniam, and H.-A. Choi, "On Double-Link Failure Recovery in WDM Optical Networks," in Proc. IEEE INFOCOM, New York, NY, June 2002.

[21] M. Clouqueur and W. Grover, "Mesh-restorable networks with enhanced dual-failure restorability properties," in Proc. SPIE OPTICOMM, Boston, MA, July 2002.

[22] W. He, M. Sridharan and A. K. Somani, "Capacity Optimization for Surviving Double-Link Failures in Mesh-Restorable Optical Networks," in Proc. SPIE OPTICOMM, Boston, MA, July 2002.

[23] J. Doucette and W. D. Grover, "Capacity Design Studies of SpanRestorable Mesh Networks with Shared-Risk Link Group (SRLG) Effects," in Proc. SPIE OPTICOMM, Boston, MA, July 2002.

[24] K.-C. Lee and V. Li, "A Wavelength Rerouting Algorithm in Wide-Area All-Optical Networks," IEEE/OSA Journal of Lightwave Technology, vol. 14, no. 6, pp. 1218-1229, June 1996.

[25] G. Mohan and C. S. R. Murthy, "A Time Optimal Wavelength Rerouting Algorithm for Dynamic Trafic in WDM Networks," IEEE/OSA Journal of Lightwave Technology, vol. 17, no. 3, pp. 406-417, Mar. 1999.

[26] D. Banerjee and B. Mukherjee, "Wavelength Routed Optical Networks: Linear Formulation, Resource Budget Tradeoffs and a Reconfiguration Study," IEEE/ACM Transactions on Networking, vol. 8, no. 5, pp. 598607, Oct. 2000.

[27] V. Anand and C. Qiao, "Static versus Dynamic Establishment of Protection Paths in WDM Networks, Part I," Journal on High Speed Networks, vol. 10, no. 4, pp. 317-327, Nov. 2001.

[28] H. Zang and B. Mukherjee, "Connection Management for Survivable Wavelength-Routed WDM Mesh Networks," Optical Networks Magazine, vol. 2, no. 4, pp. 17-28, July 2001

[29] "Ultra-low Latency, Multi-protocol Optical Routers for the NGI," http://www.darpa.mil/ipto/psum2000/K210-0.html, Nov. 2000.

[30] N.-F. Huang, G.-H. Liaw, and C.-P. Wang, "A novel all-optical transport network with time-shared wavelength channels," IEEE Journal on Selected Areas in Communications, vol. 18, no. 10, pp. 1863-1875, Oct. 2000. 